\title{
A HELLY-TYPE THEOREM FOR THE DIMENSION OF THE KERNEL OF A STARSHAPED SET
}

\author{
MARILYN BREEN
}

\begin{abstract}
This study will investigate the dimension of the kernel of a starshaped set, and the following result will be obtained: Let $S$ be a compact set in some linear topological space $L$. For $1<k<n$, the dimension of ker $S$ is at least $k$ if and only if for some $\varepsilon>0$ and some $n$-dimensional flat $F^{n}$ in $L$, every $f(n, k)$ points of $S$ see via $S$ a common $k$-dimensional neighborhood in $F^{n}$ having radius $\varepsilon$. The number $f(n, k)$ is defined inductively as follows:
\end{abstract}

$$
\begin{aligned}
& f(2,1)=4, \\
& f(n, k)=f(n-1, k)+n+2 \text { for } 3<n \text { and } 1<k<n-1, \\
& f(n, n)=n+1 .
\end{aligned}
$$

1. Introduction. We begin with some preliminary definitions: Let $S$ be a subset of some linear topological space. For points $x$ and $y$ in $S$, we say $x$ sees $y$ via $S$ if and only if the corresponding segment $[x, y]$ lies in $S$. The set $S$ is said to be starshaped if and only if there is some point $p$ in $S$ such that, for every $x$ in $S, p$ sees $x$ via $S$. The set of all such points $p$ is called the (convex) kernel of $S$, denoted ker $S$.

A well-known theorem by Krasnosel'skii [3] states that if $S$ is a compact set in some linear topological space $L$, then $S$ is starshaped if and only if for some $n$-dimensional flat $F^{n}$ in $L$, every $n+1$ points of $S$ see via $S$ a common point of $F^{n}$. It would be interesting to obtain an analogue of Krasnosel'skiirs theorem for sets whose kernel is at least $k$-dimensional, $1<k<n$. An appropriate analogue concerning the dimension of the kernel of a planar set was given in [1], and in this work, we develop a technique to extend these planar results to higher dimensions.

As in [1], the following familiar terminology will be used: Throughout the paper, conv $S$, cl $S$, int $S$, rel int $S$, bdry $S$, and $\operatorname{ker} S$ will denote the convex hull, closure, interior, relative interior, boundary, and kernel, respectively, of the set $S$. Further, if $S$ is convex, $\operatorname{dim} S$ will represent the dimension of $S$.

\section{Proof of the theorem.}

THEOREM. Let $S$ be a compact subset of some linear topological space L. For $0 \leqslant k \leqslant n$, the dimension of $\operatorname{ker} S$ is at least $k$ if and only if for some $\varepsilon>0$ and some $n$-dimensional flat $F^{n}$ in $L$, every $f(n, k)$ points of $S$ see via $S$ a

Received by the editors March 17, 1978.

AMS (MOS) subject classifications (1970). Primary 52A30, 52A35; Secondary 52A20. 
common $k$-dimensional neighborhood in $F^{n}$ having radius $\varepsilon$. The number $f(n, k)$ is defined inductively as follows:

$$
\begin{aligned}
& f(2,1)=4 \\
& f(n, k)=f(n-1, k)+n+2 \text { for } 3 \leqslant n \text { and } 1<k<n-1, \\
& f(n, n)=n+1
\end{aligned}
$$

Proof. The necessity of the condition is obvious, so we need only establish its sufficiency. We consider separately the cases for $k=n$ and $1<k<n-$ 1 , and the proof is accomplished by Lemmas 1 and 2 below.

LEMMA 1. If every $f(n, n)=n+1$ points of $S$ see a common $n$-dimensional neighborhood in $F^{n}$ having radius $\varepsilon$, then $\operatorname{ker} S$ contains an $n$-dimensional neighborhood having radius $\varepsilon$.

Proof of LEMMA 1. For convenience of notation, for each $y$ in $S$ we define the set $S_{y}=\left\{x: x \in F^{n}\right.$ and $\left.[x, y] \subseteq S\right\}$, and define $V_{y}=\{x: x$ is the center of an $\varepsilon$-neighborhood in $\left.S_{y}\right\}$. From our hypothesis, every $n+1$ of the compact convex sets conv $V_{y}$ have a nonempty intersection, so by Helly's theorem in $F^{n}$,

$$
\bigcap\left\{\operatorname{conv} V_{y}: y \text { in } S\right\} \neq \varnothing \text {. }
$$

Select a point $z$ in $\cap\left\{\operatorname{conv} V_{y}\right\} \subseteq \bigcap\left\{\operatorname{conv} S_{y}\right\}$. Using Carathéodory's theorem, since $z \in \operatorname{conv} V_{y}$ for every $y$ in $S, z$ is in the convex hull of $n+1$ or fewer points in $V_{y}$ for each $y$. Furthermore, since every point of $V_{y}$ is the center of an $\varepsilon$-neighborhood in $S_{y}$, an easy geometric argument reveals that $z$ itself must be the center of an $\varepsilon$-neighborhood in conv $S_{y}$ for every $y$. However, by adapting a standard proof of Krasnosel'skiîs theorem [2, Theorem 2.5], we see that

$$
\bigcap\left\{\operatorname{conv} S_{y}\right\}=\operatorname{ker} S \cap F^{n} \text {. }
$$

Hence $z$ is the center of an $\varepsilon$-neighborhood in $\operatorname{ker} S \cap F^{n}$, and ker $S$ contains an $n$-dimensional $\varepsilon$-neighborhood, the desired result for Lemma 1 .

LEMMA 2. Let $2 \leqslant n$ and $1 \leqslant k \leqslant n-1$. If every $f(n, k)$ points of $S$ see a common $k$-dimensional neighborhood in $F^{n}$ having radius $\varepsilon$, then $\operatorname{dim} \operatorname{ker} S>$ $k$.

Proof of Lemma 2. We induct on the dimension of the flat $F^{n}$. If $n=2$, the number $f(2,1)=4$ follows easily from the proof of [1, Theorem 1]. Hence, assume that the lemma is true for natural numbers less than $n, 3<n$, to prove for $n$. Since $n+1 \leqslant f(n, k)$, by the proof of Krasnosel'skiirs theorem, there is some point $z$ in $\operatorname{ker} S \cap F^{n}$, and without loss of generality, let $z \in \operatorname{rel} \operatorname{int}\left(\operatorname{ker} S \cap F^{n}\right)$. Let $D$ denote the closed $n$-dimensional $\varepsilon$-ball in $F^{n}$ centered at $z$.

By hypothesis, to each $f(n, k)$-member subset $T$ of $S$ we may associate at least one $k$-dimensional $\varepsilon$-neighborhood $N_{T}$ in $F^{n}$ seen by $T$. Since $z \in \operatorname{ker} S$, standard arguments reveal that $\operatorname{conv}(N \cup\{t, z\}) \subseteq S$ and hence each point 
of $T$ sees $\operatorname{conv}(N \cup\{z\})$ via $S$. Furthermore, since the radius of $N$ is $\varepsilon$, bdry $D \cap \operatorname{conv}(N \cup\{z\}) \neq \varnothing$. To each $y$ in $S$ we associate the family $\Re_{y}$ consisting of all $k$-dimensional $\varepsilon$-neighborhoods $N$ in $F^{n}$ seen by $y$. Let $S_{y}=\left\{x: x\right.$ in $F^{n}$ and $\left.[x, y] \subseteq S\right\}$ and define $A_{y}=\{x: x \in D \cap \operatorname{conv}(N \cup$ $\{z\})$ for some $N$ in $\left.\Re_{y}\right\}$. Finally, let $B_{y}=A_{y} \cup(\operatorname{ker} S \cap D)$. Clearly $B_{y} \subseteq$ $S_{y}$ for each $y$, so $\cap\left\{\operatorname{conv} B_{y}\right\} \subseteq \cap\left\{\operatorname{conv} S_{y}\right\}$. Also, by the proof of Krasnosel'skii’s theorem, $\cap\left\{\right.$ conv $\left.S_{y}\right\} \subseteq \operatorname{ker} S$, so $\cap\left\{\right.$ conv $\left.B_{y}\right\} \subseteq \operatorname{ker} S$.

If for some fixed $r>0$, each set conv $B_{y}$ contains an $n$-dimensional $r$-neighborhood of $z$, then $\operatorname{dim} \operatorname{ker} S \geqslant n$ and the proof is finished. Otherwise, by our choice of $z$, clearly $\operatorname{dim}\left(\operatorname{ker} S \cap F^{n}\right)<n$. For each $y$ in $S$ we select a hyperplane $H_{y}$ in $F^{n}$ supporting conv $B_{y}$ for which the distance $d(y)$ from $z$ to $H_{y}$ is minimal. Let $\left(H_{y}\right)_{1}$ and $\left(H_{y}\right)_{2}$ denote the corresponding open halfspaces in $F^{n}$, labeled so that conv $B_{y} \subseteq \operatorname{cl}\left(H_{y}\right)_{1}$. Certainly either some $d(y)$ is zero or some sequence of $d(y)$ numbers converges to zero. Hence there is a corresponding sequence of hyperplanes $\left\{H_{y_{m}}\right\}=\left\{H_{m}\right\}$ (possibly constant) which converges to a hyperplane $H$ containing $z$. We may label the halfspaces $H_{1}$ and $H_{2}$ determined by $H$ so that a subsequence of $\left\{\left(H_{m}\right)_{1}\right\}$ converges to $H_{1}$. Hence, without loss of generality, assume that $\left\{\operatorname{cl}\left(H_{m}\right)_{1}\right\}$ converges to cl $H_{1}$.

Notice that $H$ contains ker $S \cap F^{n}$ : Since $z \in H \cap \operatorname{rel} \operatorname{int}\left(\operatorname{ker} S \cap F^{n}\right)$, either $\operatorname{ker} S \cap F^{n} \subseteq H$ or $H$ separates points of $\operatorname{ker} S \cap F^{n}$. If the latter occurred, then for $m$ sufficiently large, $H_{y_{m}}$ would also separate points of ker $S \cap F^{n}$, impossible since $\operatorname{ker} S \cap D \subseteq B_{y} \subseteq \operatorname{cl}\left(H_{y_{m}}\right)_{1}$ for each $y_{m}$.

To complete the proof, we will show that every $f(n-1, k)$ points of $S$ see a common $k$-dimensional $\varepsilon$-neighborhood in the $(n-1)$-flat $H$. First, we establish that every $(f(n, k)-1)$-member subset $T$ of $S$ sees a common $k$-dimensional $\varepsilon$-neighborhood in cl $H_{1}$ : For each hyperplane $H_{y}$ and each neighborhood $N$ in $\Re_{y}, N$ is necessarily disjoint from $\left(H_{y}\right)_{2}$. Therefore, each $k$-dimensional $\varepsilon$-neighborhood seen by $T \cup\{y\}$ necessarily lies in $\operatorname{cl}\left(H_{y}\right)_{1}$. Since a sequence of these closed halfspaces converges to $\mathrm{cl} H_{1}$, standard arguments reveal that the points of $T$ see a common $k$-dimensional $\varepsilon$-neighborhood in cl $H_{1}$, and the preliminary result is obtained.

If every $f(n-1, k)$ points of $S$ see a common $k$-dimensional $\varepsilon$-neighborhood in $H$, this portion of our argument is complete. Hence we assume that the result fails for some $(f(n-1, k))$-member subset $T^{\prime}$, to reach a contradiction. Since

$$
f(n-1, k)=f(n, k)-n-2<f(n, k)-1,
$$

by our argument above, points of $T^{\prime}$ see a common $k$-dimensional $\varepsilon$-neighborhood in $\mathrm{cl} H_{1}$. Moreover, if no such neighborhood is in $H$, then there exists a hyperplane $J$ parallel to $H$ having the following properties: For $J_{1}$ one of the corresponding open halfspaces, $\mathrm{cl} J_{1} \subseteq H_{1}$ and every $k$ dimensional $\varepsilon$-neighborhood in $\mathrm{cl} H_{1}$ seen by $T^{\prime}$ contains some point of $\operatorname{cl} J_{1}$. (Otherwise, using the compactness of $S$, some sequence of these neighborhoods would converge to a matching neighborhood in $H$.) Since every $f(n, k)-1=f(n-1, k)+n+1$ points of $S$ see such a neighborhood in cl $H_{1}$ and $T^{\prime}$ has $f(n-1, k)$ members, this forces every $n+1$ points of $S$ to 
see a common point of $\mathrm{cl} J_{1}$. Therefore by the proof of Krasnosel'skii's theorem, cl $J_{1} \cap \operatorname{ker} S \neq \varnothing$. However, this is impossible since ker $S \cap F^{n} \subseteq$ $H$ and $\mathrm{cl} J_{1} \subseteq H_{1}$. We have a contradiction, our assumption is false, and we conclude that every $f(n-1, k)$ points of $S$ see a common $k$-dimensional $\varepsilon$-neighborhood in $H$, the desired result.

Finally, our inductive hypothesis may be applied to the $(n-1)$-flat $H$ to conclude that $\operatorname{dim} \operatorname{ker} S>k$. This completes the proof of Lemma 2 and finishes the proof of the theorem.

The following example illustrates that the number $f(n, n)$ in the theorem is best possible.

EXAMPLE 1. Let $S_{1}$ denote an $n$-simplex whose interior contains the origin. Define $S_{2}=2 S_{1}$ and let $S=\operatorname{cl}\left(S_{2} \sim S_{1}\right)$. Then for an appropriate choice of $\varepsilon>0$, every $n$ points of $S$ see via $S$ a common $n$-dimensional $\varepsilon$-neighborhood, yet ker $S=\varnothing$.

Furthermore, the number $f(2,1)$ in the theorem is best possible by [1, Example 1]. However, best values for $3 \leqslant n$ and $1 \leqslant k \leqslant n-1$ have not been established.

We conclude with two final remarks:

REMARK 1. Clearly the requirement that $S$ be compact in Theorem 1 may be replaced with the conditions that $S$ be closed and $S \cap F^{n}$ be compact. However, [1, Examples 3 and 4] may be extended to $R^{n}$ to show that unless $S \cap F^{n}$ is both closed and bounded, no finite analogue of the bound $f(n, k)$ is possible.

REMARK 2. The inductive formulas defining $f(n, k)$ in Theorem 1 may be replaced with the following sums:

$$
\begin{aligned}
& f(n, 1)=\sum\{i: 4 \leqslant i \leqslant n+2\} \quad \text { if } 2 \leqslant n, \\
& f(n, k)=k+1+\sum\{i: k+3 \leqslant i \leqslant n+2\} \text { if } 2<k<n-1, \\
& f(n, n)=n+1 .
\end{aligned}
$$

\section{REFERENCES}

1. Marilyn Breen, The dimension of the kernel of a planar set, Pacific J. Math. (to appear).

2. L. Danzer, B. Grünbaum and V. Klee, Helly's theorem and its relatives, Convexity, Proc. Sympos. Pure Math., vol. 7, Amer. Math. Soc., Providence, R. I., 1962, pp. 101-180.

3. M. A. Krasnosel'skii, Sur un critère pour qu'un domain soit étoilé, Math. Sb. 19 (61) (1946), 309-310.

Department of Mathematics, University Of OKLahoma, Norman, OkLahoma 73019 